\title{
Probing the Ubiquitin Landscape
}

\author{
Monique P. Mulder ${ }^{1 *}$, Zhihao Zhuang ${ }^{2}$, Lei Liư ${ }^{3}$, Benedikt M. Kessler ${ }^{4}$, Huib Ovaa ${ }^{1}$ \\ ${ }^{1}$ Leiden University Medical Center, Netherlands, ${ }^{2}$ University of Delaware, United States, ${ }^{3}$ Tsinghua- \\ Peking Center for Life Sciences, School of Life Sciences, Tsinghua University, China, ${ }^{4}$ University of \\ Oxford, United Kingdom \\ Submitted to J ournal: \\ Frontiers in Chemistry \\ Specialty Section: \\ Chemical Biology \\ Article type: \\ Editorial Article \\ Manuscript ID: \\ 553741 \\ Received on: \\ 20 Apr 2020 \\ Frontiers website link: \\ www. frontiersin.org
}




\section{Conflict of interest statement}

The authors declare that the research was conducted in the absence of any commercial or financial relationships that could be construed as a potential conflict of interest

\section{Author contribution statement}

All authors listed have made a substantial, direct and intellectual contribution to the work, and approved it for publication.

\section{Keywords}

Ubiquitin(-like), activity-based probes, toolbox development, applications, inhibitors, Proteomics

\section{Contribution to the field}

We believe that our Probing the Ubiquitin Landscape Research Topic exemplifies the multidisciplinary nature of research in the ubiquitin field. As the field advances, we increase our understanding by interconnected research strategies, thereby allowing development of therapeutics as exemplified in this body of work. 


\title{
Probing the Ubiquitin Landscape
}

\author{
Monique P.C. Mulder ${ }^{1}$, Zhihao Zhuang ${ }^{2}$, Lei Liu ${ }^{3}$, Benedikt M. Kessler ${ }^{4}$, Huib Ovaa ${ }^{1}$
}

\author{
${ }^{1}$ Department of Cell and Chemical Biology, Oncode Institute, Leiden University Medical Centre, Leiden, Netherlands \\ 2Department of Chemistry and Biochemistry, 214A Drake Hall, University of Delaware, Newark, Delaware 19716, \\ United States. \\ ${ }^{3}$ Tsinghua-Peking Center for Life Sciences, MOE Key Laboratory of Bioorganic Phosphorus Chemistry \& Chemical \\ Biology, Center for Synthetic and Systems Biology, Department of Chemistry, Tsinghua University, Beijing, 100084, \\ China. \\ ${ }^{4}$ Nuffield Department of Medicine, University of Oxford, Oxford, United Kingdom.
}

Since the pioneer work of Hershko, Ciechanover and their colleagues 40 years ago, our understanding of one of the most complex and widespread signaling networks in biology has evolved greatly through genetic, proteomic, biochemical and cell biological studies (Kliza and Husnjak). In this Research Topic we present a salient collection of original research, methods and review articles that cover promising, recent, and novel research trends in the ubiquitin and ubiquitin-like field.

Protein ubiquitination is a powerful post-translational modulator (PTM) of cellular functions and occurs in various forms, which are usually referred to as the "Ub code". Modification by ubiquitin (Ub) to a target protein is tightly controlled by the action of hundreds of regulatory enzymes employed in specific combinations of three enzyme classes: Ub activating enzyme E1, conjugating enzyme E2, and Ub ligase E3. To counterbalance ubiquitination, it can be removed from its substrates by deubiquitinating enzymes (DUBs). Modification by a single Ub moiety (monoubiquitination) is the most abundant Ub modification. However, after Ub is transferred to the $\varepsilon$-amino group of a target Lys, any of the eight amino groups of Ub (Met1, Lys6, Lys11, Lys27, Lys29, Lys33, Lys48, Lys63) can be attached to the C terminus of another Ub to form polyUb chains of variable length, linkage type and configuration (homo- and heterotypic/branched Ub chains). Even though the functional significance of cellular Ub modifications (such as Lys48- and Lys63linked ubiquitination) is largely known, the biological significance of other homotypic polyUb chains, collectively called atypical Ub chains, is still far from being fully understood. Van Huizen et al. comprehensively review the role of atypical ubiquitin chains in the regulation of intracellular antiviral innate immune signaling pathways. Dittmar et al. review a distinct type of ubiquitination, referred to as linear (Met1-linked) ubiquitination, a transient and spatially regulated modification, making their detection and quantification challenging.

Next to ubiquitin, a vast number of ubiquitin-like proteins (e.g. SUMO, Nedd8, ISG15, Ufm1, Fat10) can also be attached to proteins increasing the complexity of and fine-tuning cellular responses even further. Keiten-Schmitz et al. review the role of SUMO chains in chromatin dynamics and genome stability networks, whereas Fernandez et al. describe ISGylation as well as strategies targeting this PTM for therapeutic applications. Intriguingly, unlike most PTMs such as 
phosphorylation or acetylation, ubiquitination can be highly customized through further posttranslational modification of ubiquitin itself, thereby coding for distinct cellular outcomes which either alter the originally encrypted message or encode a completely new one. In addition to ubiquitination, $\mathrm{Ub}$ molecules can be modified by acetylation, phosphorylation, succinylation, ADPribosylation, deamidation, and Ub-like proteins (UbL). The cross-functionality introduced by the latter hybrid Ub/UbL chains is discussed by Perez Berrocal et al.

Considering the impact of ubiquitination on the regulation of a vast array of fundamental biological processes, dysregulation of this intrinsic process gives rise to numerous diseases ranging from autoimmunity, cancer, and neurodegenerative diseases such as Alzheimer and Huntington's disease (Sap et al.). Defects in the ubiquitin-proteasome system (UPS) - a central player in protein quality control facilitating elimination of misfolded or otherwise aberrant proteins, maintaining protein homeostasis in cells - including when the activity of a given E3 ligase is hampered, have been the focus of many studies during the last few years. Understanding the mechanism of action, as well as identifying which substrates are regulated by each E3 at different developmental stages and cell types, will provide invaluable knowledge that might contribute to develop therapeutic strategies to treat these diseases. Garcia-Barcena et al. review the generation and usage of E3 mutants which have revealed unexpected and important lessons about the complexity of this family of enzymes. As the E3 enzymes exhibit different substrate specificity, determining which E3 enzyme maps to which substrate is crucial for our understanding of this complex intrinsic network. Salas-Lloret et al. describe an improved TULIP2 methodology facilitating mass spectrometry towards the identification of E3-substrate networks.

While proteasome inhibition is in clinical use for the treatment of hematopoietic malignancies, stimulation of the UPS has been proposed as a potential therapeutic strategy for various neurodegenerative disorders. High-throughput screens (HTS) using genetic approaches or compound libraries are powerful tools to identify therapeutic intervention points and novel drugs. Franklin and Pruneda describe a new assay, UbiReal, that make use of fluorescence polarization to monitor all stages of Ub conjugation and deconjugation in real time, making it an excellent candidate for HTS of activity modulators as well as a valuable tool for basic research into the mechanisms of ubiquitin regulation. Next to assays that measure specific enzyme activities of components of the UPS as exemplified by Franklin and Pruneda, reporter substrates provide us with a view of the general functional status of the UPS in cells. Gierisch et al. discuss several reporter substrates for the UPS and their application in the identification of key players in the pursuit for novel therapeutics. More recently technologies have been established that induce targeted protein degradation by chimeric small molecules as reviewed by Naito et al. These technologies, such as Proteolysis Targeting Chimeras (PROTACs), hijack the cellular machinery for ubiquitination thereby subjecting the ubiquitinated proteins to proteasomal degradation. This has promoted several drug development research programs because "undruggable targets" can now be degraded by targeted protein degradation. 
With an increased focus on the development of inhibitors of ubiquitin(-like) system components, characterization of their dynamics is imminent. Understanding the mechanisms and functional relevance of ubiquitin as a signaling system requires the generation of antibodies or alternative reagents that specifically detect ubiquitin in a site-specific manner. Kruijsbergen et al. describe the challenges and a strategy for the development of site-specific ubiquitin antibodies. Together with advances in synthetic strategies for generating ubiquitin, the development of a plethora of ubiquitin assay reagents and numerous activity-based probes (ABPs), enabled the study of enzymes involved in the complex system of ubiquitination. ABPs react covalently with an active site residue to report on specific enzyme activity, and thus represent a powerful method to evaluate cellular and physiological enzyme function and dynamics. Taylor et al. present the developments made in the 'traditional' ubiquitin based ABPs, whereas Conole et al. discuss recent developments in cell permeable small molecule ABPs. With these advances in the ABP field, activity based protein profiling has emerged as a powerful technique to study these important enzymes as exemplified by the work of Pinto-Fernández et al. Mass spectrometry technology has now reached the capacity to cover the proteome with enough depth to interrogate entire biochemical pathways including those that contain DUBs and E3 ligase substrates. PintoFernández et al. have combined advanced mass spectrometry technology with propargylic-based ubiquitin ABPs to reveal the proportion of active cellular DUBs adding another layer of information in addition to their endogenous expression levels.

Further understanding of the ubiquitin signaling pathway includes the knowhow on how polyUbspecific interacting proteins recognize different polyUb chains. These interacting proteins often contain a specific ubiquitin-binding domain (UBD) that can bind to specific polyUb chains, leading to different cellular responses. Hameed et al. report the synthesis of diUb chains, fully $15 \mathrm{~N}$-labeled on the distal (N-terminal) Ub and demonstrate their applicability as tools for structural insights into the selective recognition of a unique UBD for a diUb linkage.

We believe that our Probing the Ubiquitin Landscape Research Topic exemplifies the multidisciplinary nature of research in the ubiquitin field. As the field advances, we increase our understanding by interconnected research strategies, thereby allowing development of therapeutics as exemplified in this body of work.

\section{AUTHOR CONTRIBUTIONS}

All authors listed have made a substantial, direct and intellectual contribution to the work, and approved it for publication. 